\title{
Large-scale instabilities of helical flows
}

\author{
Alexandre CAmeron Alexandros Alexakis ${ }^{7}$ and Marc-Étienne Brachet \\ Laboratoire de Physique Statistique, École Normale Supérieure, \\ PSL Research University; Université Paris Diderot Sorbonne Paris-Cité; Sorbonne \\ Universités UPMC Univ Paris 06; CNRS; 24 rue Lhomond, 75005 Paris, France
}

(Dated: March 7, 2022)

\begin{abstract}
Large-scale hydrodynamic instabilities of periodic helical flows are investigated using 3D Floquet numerical computations. A minimal three-modes analytical model that reproduce and explains some of the full Floquet results is derived. The growth-rate $\sigma$ of the most unstable modes (at small scale, low Reynolds number $R e$ and small wavenumber $q$ ) is found to scale differently in the presence or absence of anisotropic kinetic alpha $(A K A)$ effect. When an $A K A$ effect is present the scaling $\sigma \propto q R e$ predicted by the $A K A$ effect theory [U. Frisch, Z. S. She, and P. L. Sulem, Physica D: Nonlinear Phenomena 28, 382 (1987)] is recovered for $R e \ll 1$ as expected (with most of the energy of the unstable mode concentrated in the large scales). However, as Re increases, the growth-rate is found to saturate and most of the energy is found at small scales. In the absence of $A K A$ effect, it is found that flows can still have large-scale instabilities, but with a negative eddy-viscosity scaling $\sigma \propto \nu\left(b R e^{2}-1\right) q^{2}$. The instability appears only above a critical value of the Reynolds number $R e^{c}$. For values of $R e$ above a second critical value $R e_{S}^{c}$ beyond which small-scale instabilities are present, the growth-rate becomes independent of $q$ and the energy of the perturbation at large scales decreases with scale separation. A simple two-modes model is derived that well describes the behaviors of energy concentration and growth-rates of various unstable flows. In the non-linear regime (at moderate values of $R e$ ) and in the presence of scale separation, the forcing scale and the largest scales of the system are found to be the most dominant energetically.
\end{abstract}

PACS numbers: 47.20.-k,47.11.St,47.11.Kb,47.15.Fe,

\section{INTRODUCTION}

Hydrodynamic instabilities are responsible for the frequent encounter of turbulence in nature. Although instabilities are connected to the onset of turbulence and the generation of small scales, in many situation, instabilities are also responsible for the formation of large-scale structures. In such situations, flows of a given coherence length-scale are unstable to larger scale perturbations transferring energy to these scales. A classical example of a large-scale instability is the $\alpha$-effect [1, 2] in magneto-hydrodynamic (MHD) flows to which the origin of large-scale planetary and solar magnetic field is attributed. In $\alpha$-dynamo theory, small-scale helical flows self-organize to generate magnetic fields at the largest scale of the system.

While large-scale instabilities have been extensively studied for the dynamo problem, limited attention has been drawn to large-scale instabilities of the pure hydrodynamic case. Hence, most direct numeric simulations (DNS) and turbulence experiments are designed so that the energy injection scale $\ell$ is close to the domain size $L$. This allows to focus on the forward energy cascade and the formation of the Kolmogorov spectrum [3]. Scales larger that the forcing scale, where no energy cascade is present, are expected [4, 5] to reach a thermal equilibrium with a $k^{2}$ spectrum [6 9]. Recent studies, using

\footnotetext{
* alexandre.cameron@ens.fr

$\dagger$ alexakis@lps.ens.fr

$\ddagger$ brachet@physique.ens.fr
}

(hyper-viscous) simulations of turbulent flows randomly forced at intermediate scales [10], have shown that the energy spectrum at large scales deviates from the thermal equilibrium prediction and forms a strong peak at the largest scale of the system. A possible explanation for this intriguing result is that a large-scale instability is present.

In pure hydrodynamic flows, the existence of largescale instabilities has been known for some time. An asymptotic expansion based on scale separation was used in [11, 12] to demonstrate the existence of a mechanism similar to the MHD $\alpha$-dynamo called the anisotropickinetic-alpha $(A K A)$ instability. The $A K A$ instability is present in a certain class of non-parity-invariant, timedependent and anisotropic flows. It appears for arbitrary small values of the Reynolds number and leads to a growth-rate $\sigma$ proportional to the wavenumber $q$ of the unstable mode: $\sigma \propto q$. However, the necessary conditions for the presence of the $A K A$ instability are stricter than those of the $\alpha$-dynamo. Thus, most archetypal flows studied in the literature do not satisfy the $A K A$ conditions for instability. This, however, does not imply that the large scales are stable since other mechanisms may be present.

In the absence of an $A K A$-effect higher-order terms in the large-scale expansion may lead to a so-called eddyviscosity effect [13]. This eddy-viscosity can be negative and thus produce a large-scale instability [14, 15]. The presence of a negative eddy-viscosity instability appears only above a critical value of the Reynolds number. It results in a weaker growth-rate than the $A K A$-effect, proportional to the square of the wavenumber of the unsta- 
ble mode $\sigma \propto q^{2}$. Furthermore, the calculations of the eddy-viscosity coefficient can be much more difficult than those of the $A K A \alpha$ coefficient. This difficulty originates on the order at which the Reynolds number enters the expansion as we explain below.

In the present paper, the Reynolds number is defined as $R e \equiv U_{r m s} \ell / \nu$ where $U_{r m s}$ is the root mean square value of the velocity and $\nu$ is the viscosity. Note that we have chosen to define the Reynolds number based on the energy injection scale $\ell$. An alternative choice would be to use the domain length scale $L$ which would lead to the large-scale Reynolds number that we will denote as $R e^{L}=U L / \nu=(L / \ell) R e$. For the $A K A$ effect, the large-scale Reynolds number $R e^{L}$ is large, while the Reynolds number $R e$, based on the forcing scale $\ell$, is small. This allows to explicitly solve for the small-scale behavior and obtain analytic results. This is not possible for the eddy-viscosity calculation where there are two regimes to consider. Either the Reynolds numbers is small and the eddy-viscosity only provides a small correction to the regular viscosity, or the Reynolds numbers is large and the inversion of an advection operator is needed. This last case can be obtained analytically only for very simple one dimensional shear flows [14, 15].

To illustrate the basic mechanisms involved in such multi-scale interactions, we depict in fig. 1 a toy model demonstrating the main ideas behind these instabilities. This toy model considers a driving flow, $\boldsymbol{U}$ at wavenumber $\mathbf{K} \sim 1 / \ell$, that couples to a small amplitude largescale flow, $\boldsymbol{v}_{\boldsymbol{q}}$ at wavenumber $\mathbf{q} \sim 1 / L$ with $|\mathbf{q}| \ll|\mathbf{K}|$. The advection of $\boldsymbol{v}_{\boldsymbol{q}}$ by $\boldsymbol{U}$ and visa versa will then generate a secondary flow $\boldsymbol{v}_{\boldsymbol{Q}}$ at wavenumbers $\boldsymbol{Q}=\boldsymbol{K} \pm \boldsymbol{q}$. This small-scale perturbation in turn couples to the driving flow and feeds back the large-scale flow. If this feedback is constructive enough to overcome viscous dissipation, it will amplify the large-scale flow and this process will lead to an exponential increase of $\boldsymbol{v}_{\boldsymbol{q}}$ and $\boldsymbol{v}_{\boldsymbol{Q}}$. This toy model has most of the ingredients required for the instabilities to occur.

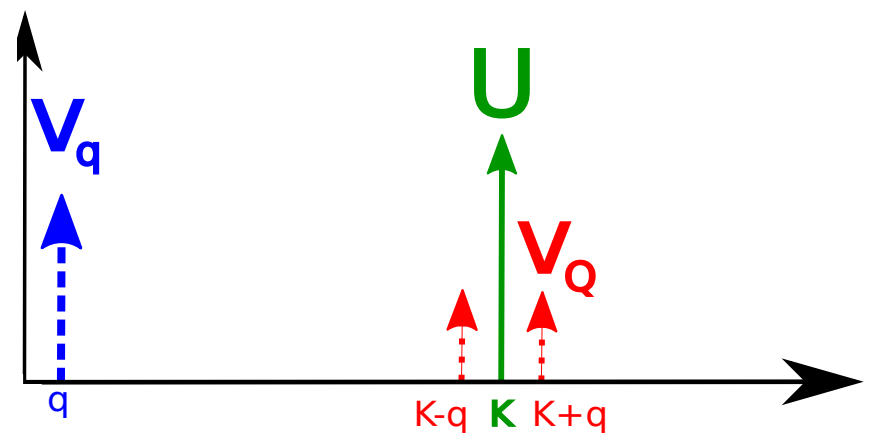

FIG. 1: (Color online) Sketch of the three-modes model. $U$ represents the small-scale driving flow of wavenumber $K$ (full arrow), $v_{q}$ is the large-scale perturbation of wavevector $q$ (dashed arrow) and $v_{Q}$ is the small scale perturbation of wavevector $Q=K \pm q$ (doted arrow).

In order to study large-scale instabilities, they must be isolated from other small-scale competing instabilities that might coexist. This can be achieved by using Floquet theory [16] (also referred as Bloch theory in quantum mechanics [17]). Indeed, Floquet theory can track modes with large and small spatial periodicity separately. In what follows, we use direct numerical simulations (DNS) in the Floquet framework to study different flows, either in the presence of the $A K A$ effect using the flow introduced in 11] or in the absence of $A K A$ effect using the equilateral $A B C$ flow $(\mathrm{A}=\mathrm{B}=\mathrm{C})$ [18] and the Roberts flow [19]. Our study extends to values of $R e$ and $\ell / L$ beyond the range of validity of the asymptotic expansions. Finally, we compare the results of Floquet DNS to those of full Navier-Stokes DNS.

\section{METHODS}

\section{A. Navier-Stokes}

Our starting point is the incompressible Navier-Stokes equation in the periodic $[0,2 \pi L]^{3}$-cube:

$$
\partial_{t} \boldsymbol{V}=\boldsymbol{V} \times \boldsymbol{\nabla} \times \boldsymbol{V}-\boldsymbol{\nabla} P+\nu \Delta \boldsymbol{V}+\boldsymbol{F},
$$

with $\boldsymbol{\nabla} \cdot \boldsymbol{V}=0$ and where $\boldsymbol{V}, \boldsymbol{F}, P$ and $\nu$ denote the velocity field, the forcing field, the generalized pressure field and the viscosity coefficient, respectively. The geometry imposes that all fields be $2 \pi L$-periodic. We further assume that the forcing has a shorter spatial period $2 \pi \ell$ with $L / \ell$ an arbitrary large integer. We denote the wavenumber of this periodic forcing as $\mathbf{K}$, with $K=|\mathbf{K}|=1 / \ell$ for the flows examined. If the initial conditions of $\mathbf{V}$ satisfies the same periodicity as $\boldsymbol{F}$ then this periodicity will be preserved by the solutions of the Navier-Stokes and corresponds to the preservation of the discrete symmetries $x \rightarrow x+2 \pi \ell, y \rightarrow y+2 \pi \ell$ and $z \rightarrow z+2 \pi \ell$. However, these solutions can be unstable to arbitrary small perturbations that break this symmetry and grow exponentially. To investigate the stability of the periodic solutions, we decompose the velocity and pressure field in a driving flow and a perturbation component:

$$
\boldsymbol{V}=\boldsymbol{U}+\boldsymbol{v}, \quad P=P_{\boldsymbol{U}}+P_{\boldsymbol{v}}
$$

where $\boldsymbol{U}$ denotes the driving flow that has the same periodicity as the forcing $2 \pi \ell$ and $\boldsymbol{v}$ is the velocity perturbation. The linear stability analysis amounts to determining the evolution of small amplitude perturbations so that only the first order terms in $\boldsymbol{v}$ are kept. The evolution equation of the driving flow is thus:

$$
\partial_{t} \boldsymbol{U}=\boldsymbol{U} \times \boldsymbol{\nabla} \times \boldsymbol{U}-\boldsymbol{\nabla} P_{\boldsymbol{U}}+\nu \Delta \boldsymbol{U}+\boldsymbol{F} .
$$

The remaining terms give the linearized Navier-Stokes equation for the perturbation:

$$
\partial_{t} \boldsymbol{v}=\boldsymbol{U} \times \boldsymbol{\nabla} \times \boldsymbol{v}+\boldsymbol{v} \times \boldsymbol{\nabla} \times \boldsymbol{U}-\boldsymbol{\nabla} p_{\boldsymbol{v}}+\nu \Delta \boldsymbol{v},
$$


The two pressure terms enforce the incompressibility conditions $\boldsymbol{\nabla} \cdot \boldsymbol{U}=0$ and $\boldsymbol{\nabla} \cdot \boldsymbol{v}=0$. The $\mathbf{U}$ flow is not necessarily a laminar flow (but respects $2 \pi \ell$ periodicity). In general, the linear perturbation $\boldsymbol{v}$ does not only consist of modes that break the periodicity of the forcing. Linear unstable modes respecting the periodicity may also exist: they correspond to small-scale instabilities. We show how these modes can be distinguished from periodicitybreaking large-scale modes in the following section devoted to Floquet analysis.

\section{B. Floquet Analysis}

Studying large-scale flow perturbations with a code that solves the full Navier-Stokes equation requires considerable computational power as resolution of all scales from domain size $L$ to the smallest viscous scales $\ell_{\nu} \ll \ell$ must be achieved. This is particularly difficult in our case where scale separation $\ell \ll L$ is required. In order to overcome this limitation, we adopt the Floquet framework [16]. In Floquet theory, the velocity perturbation can be decomposed into modes that are expressed as the product of a complex harmonic wave, $e^{i \boldsymbol{q} \cdot \mathbf{r}}$, multiplied by a periodic vector field $\tilde{\boldsymbol{v}}(\boldsymbol{r}, t)$ with the same periodicity $2 \pi \ell$ as that of the driving flow:

$$
\boldsymbol{v}(\boldsymbol{r}, t)=\tilde{\boldsymbol{v}}(\boldsymbol{r}, t) e^{\imath \boldsymbol{q} \cdot \boldsymbol{r}}+\text { c.c. },
$$

and similar for the pressure,

$$
p_{\boldsymbol{v}}(\boldsymbol{r}, t)=\tilde{p}(\boldsymbol{r}, t) e^{\imath \boldsymbol{q} \cdot \boldsymbol{r}}+\text { c.c. },
$$

where c.c. denotes the complex conjugate of the previous term.

Perturbations whose values of $\mathbf{q}$ are such that at least one component is not an integer multiple of $1 / \ell$, break the periodicity of the driving flow. The perturbation field $\boldsymbol{v}$ then involves all Fourier wavenumbers of the type $\mathbf{Q}=\mathbf{q}+\mathbf{k}$, where $\mathbf{k}$ is a wavevector corresponding to the $2 \pi \ell$-periodic space dependence of $\tilde{\boldsymbol{v}}$. We restrict the study to values of $q=|\mathbf{q}|$ satisfying $0<q \leq K$. For finite domain sizes $\mathbf{q}$ is a discrete vector with $q \geq 1 / L$, while for infinite domain sizes $\boldsymbol{q}$ can take any arbitrarily small value. In the limit $q / K \ll 1$ the perturbation involves scales much larger than $\ell$. Therefore, scale separation is achieved without solving intermediate scales as would be required if the full Navier-Stokes equations were used. Furthermore, this framework has the advantage of isolating perturbations that break the forcing periodicity $\left(\boldsymbol{q} \ell \notin \mathbb{Z}^{3}\right)$, from other small-scale unstable modes with the same periodicity $\left(\boldsymbol{q} \ell \in \mathbb{Z}^{3}\right)$ that might also exist in the system.

A drawback of the Floquet decomposition is that some operators have somewhat more complicated expressions than in the simple periodic case. For instance, taking a derivative requires to take into account the variations of both the harmonic and the amplitude. Separating the amplitude in its real and imaginary parts $\tilde{\boldsymbol{v}}(\boldsymbol{r}, t)=\tilde{\boldsymbol{v}}^{r}+$ $\imath \tilde{\boldsymbol{v}}^{i}$, we obtain

$$
\partial_{x} \boldsymbol{v}=\left[\partial_{x} \tilde{\boldsymbol{v}}^{r}-q_{x} \tilde{\boldsymbol{v}}^{i}+\imath\left(q_{x} \tilde{\boldsymbol{v}}^{r}+\partial_{x} \tilde{\boldsymbol{v}}^{i}\right)\right] e^{\imath \boldsymbol{q} \cdot \boldsymbol{r}}+\text { c.c. },
$$

where $\partial_{x}$ denotes the $x$-derivative and $q_{x}$ denotes the $x$ component of the $\boldsymbol{q}$ wavevector.

Using eq. (4) and (7), the linearized Navier-Stokes equation can be written as a set of $3+1$ complex scalar equations:

$$
\begin{aligned}
& \partial_{t} \tilde{\boldsymbol{v}}=(\boldsymbol{\nabla} \times \boldsymbol{U}) \times \tilde{\boldsymbol{v}}+(\imath \boldsymbol{q} \times \tilde{\boldsymbol{v}}+\boldsymbol{\nabla} \times \tilde{\boldsymbol{v}}) \times \boldsymbol{U} \\
&-(\imath \boldsymbol{q}+\boldsymbol{\nabla}) \tilde{p}+\nu\left(-\boldsymbol{q}^{2}+\Delta\right) \tilde{\boldsymbol{v}}, \\
& \text { with } \quad \imath \boldsymbol{q} \cdot \tilde{\boldsymbol{v}}+\boldsymbol{\nabla} \cdot \tilde{\boldsymbol{v}}=0 .
\end{aligned}
$$

We use standard pseudo-spectral methods to solve this system of equations in the $2 \pi \ell$-periodic cube. The complex velocity field $\tilde{\boldsymbol{v}}$ is decomposed in Fourier space where derivatives are reduced to a multiplication by $\boldsymbol{\imath} \boldsymbol{k}$, where $\boldsymbol{k}$ is the Fourier wavevector. Multiplicative term are computed in real space. These methods have been implemented in the: Floquet Linear Analysis for Spectral Hydrodynamics (FLASH) code and details are given in appx. $\nabla$.

In order to find the growth-rate of the most unstable mode, we integrate eq. (8), (9), for a time long enough for a clear exponential behaviour to be observed. The growth-rate of this most unstable mode can then be measured by linear fitting. Note that this process only leads to the measurement of the fastest growing mode.

\section{Three-modes model}

Although the Floquet framework is very convenient to solve equations numerically, it does not easily yield analytic results. Rigorous results must be based on asymptotic expansions and can only be derived in the limit of small Reynolds number or for simple shear layers [14, 15]. To obtain a basic understanding of the processes involved, we will use the idea represented in the toy model of fig. 1 This model also has the major advantage of using a formalism that can easily be related to the physical aspect of the problem.

In our derivation, we only consider the evolution of the two most intense modes of the perturbation and of the driving flow. The velocity perturbation is thus decomposed as a series of velocity fields of different modes:

$$
\begin{aligned}
& \boldsymbol{v}(\boldsymbol{r}, t)=\boldsymbol{v}_{\boldsymbol{q}}(\boldsymbol{r}, t)+\boldsymbol{v}_{\boldsymbol{Q}}(\boldsymbol{r}, t)+\boldsymbol{v}_{>}(\boldsymbol{r}, t), \\
& \boldsymbol{v}_{\boldsymbol{q}}(\boldsymbol{r}, t)=\tilde{\boldsymbol{v}}(\boldsymbol{q}, t) e^{\imath \boldsymbol{q} \boldsymbol{r}}+\text { c.c. }, \\
& \boldsymbol{v}_{\boldsymbol{Q}}(\boldsymbol{r}, t)=\sum_{\|\boldsymbol{k}\|=1} \tilde{\boldsymbol{v}}(\boldsymbol{q}, \boldsymbol{k}, t) e^{\imath(\boldsymbol{q} \cdot \boldsymbol{r}+\boldsymbol{k} \cdot \boldsymbol{r})}+c . c ., \\
& \boldsymbol{v}_{>}(\boldsymbol{r}, t)=\sum_{\|\boldsymbol{k}\|>1} \tilde{\boldsymbol{v}}(\boldsymbol{q}, \boldsymbol{k}, t) e^{\imath(\boldsymbol{q} \cdot \boldsymbol{r}+\boldsymbol{k} \cdot \boldsymbol{r})}+c . c .,
\end{aligned}
$$

where $\boldsymbol{q}$ denotes the wavenumber of the large-scale modes and $\boldsymbol{Q}$ denotes the modes directly coupled to $\boldsymbol{q}$ via the 
driving flow, since $K=1$. At wavenumber $\boldsymbol{q}$, the linearized Navier-Stokes equation can be rewritten as:

$$
\partial_{t} \boldsymbol{v}_{\boldsymbol{q}}=\boldsymbol{U} \times \boldsymbol{\nabla} \times \boldsymbol{v}_{\boldsymbol{Q}}+\boldsymbol{v}_{\boldsymbol{Q}} \times \boldsymbol{\nabla} \times \boldsymbol{U}-\boldsymbol{\nabla} p_{\boldsymbol{q}}+\nu \Delta \boldsymbol{v}_{\boldsymbol{q}}
$$

Assuming that the coupling with the truncated velocity, $\boldsymbol{v}_{>}$, is negligible with respect to the coupling with the large-scale velocity, $\boldsymbol{v}_{\boldsymbol{q}}$, the linearized equation at $\boldsymbol{Q}$ reads:

$$
\partial_{t} \boldsymbol{v}_{\boldsymbol{Q}}=\boldsymbol{U} \times \boldsymbol{\nabla} \times \boldsymbol{v}_{\boldsymbol{q}}+\boldsymbol{v}_{\boldsymbol{q}} \times \boldsymbol{\nabla} \times \boldsymbol{U}-\nabla p_{\boldsymbol{Q}}+\nu \Delta \boldsymbol{v}_{\boldsymbol{Q}},
$$

where $p_{\boldsymbol{q}}$ and $p_{\boldsymbol{Q}}$ denote the pressure enforcing the incompressible conditions: $\boldsymbol{\nabla} \cdot \boldsymbol{v}_{\boldsymbol{q}}=0$ and $\boldsymbol{\nabla} \cdot \boldsymbol{v}_{\boldsymbol{Q}}=0$, respectively. The modes are represented in fig. 2

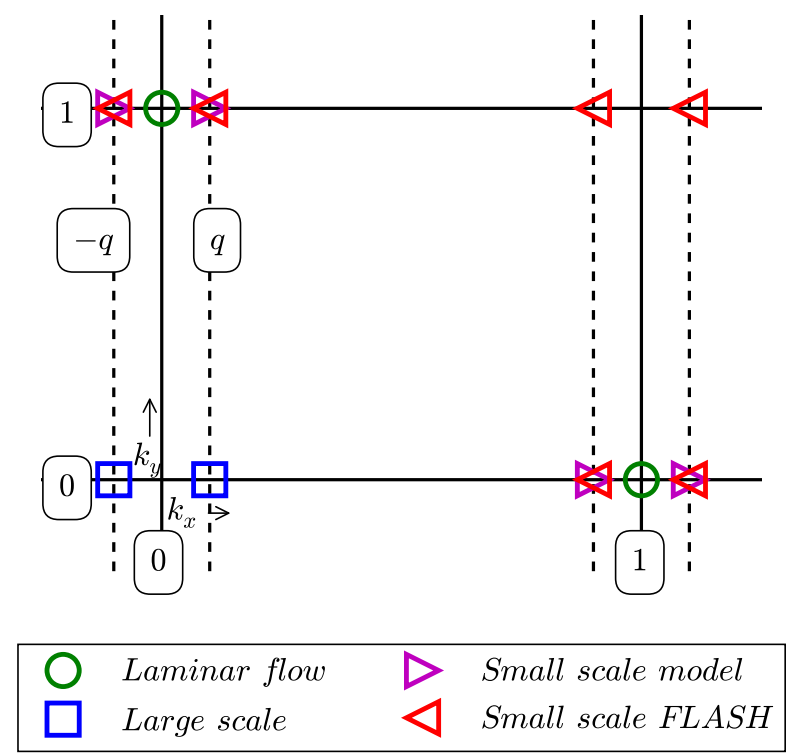

FIG. 2: Fourier modes of the Floquet decomposition used in the FLASH code and the three-modes model.

The derivation is restricted to stationary positive helical driving flows, satisfying: $\boldsymbol{U}_{\mathcal{H}}(\boldsymbol{r})=K^{-1} \nabla \times \boldsymbol{U}_{\mathcal{H}}(\boldsymbol{r})$. The problem can then be solved by making use of the vorticity fields:

$$
\omega_{q}=\nabla \times v_{q} \quad \text { and } \quad \omega_{Q}=\nabla \times v_{Q},
$$

and the adiabatic approximation: $\partial_{t} \boldsymbol{v}_{\boldsymbol{Q}} \ll \nu \Delta \boldsymbol{v}_{\boldsymbol{Q}}$. The system of equations of the three-modes model is thus:

$$
\begin{aligned}
\nu \boldsymbol{\omega}_{\boldsymbol{Q}} & =-\boldsymbol{\nabla} \times\left[\boldsymbol{U}_{\mathcal{H}} \times\left(\boldsymbol{\omega}_{\boldsymbol{q}}-K \boldsymbol{v}_{\boldsymbol{q}}\right)\right], \\
\partial_{t} \boldsymbol{\omega}_{\boldsymbol{q}} & =\boldsymbol{\nabla} \times\left[\boldsymbol{U}_{\mathcal{H}} \times\left(\boldsymbol{\omega}_{\boldsymbol{Q}}-K \boldsymbol{v}_{\boldsymbol{Q}}\right)\right]+\nu \Delta \boldsymbol{\omega}_{\boldsymbol{q}} .
\end{aligned}
$$

The greatest eigenvalue of the system, $\sigma$, gives the growth-rate of the perturbation. The growth-rate can be derived analytically for an $A B C$ large-scale flow:

$$
\begin{aligned}
& U_{x}^{A B C}=C \sin (K z)+B \cos (K y), \\
& U_{y}^{A B C}=A \sin (K x)+C \cos (K z), \\
& U_{z}^{A B C}=B \sin (K y)+A \cos (K y) .
\end{aligned}
$$

For $A=1: B=1: C=\lambda$ flows $(\lambda-A B C)$, one finds:

$$
\begin{gathered}
\sigma=\beta q^{2}-\nu q^{2} \quad \text { with } \quad \beta=b R e^{2} \nu, \\
b=\frac{1-\lambda^{2}}{4+2 \lambda^{2}} \quad \text { and } \quad R e=\frac{U}{K \nu}
\end{gathered}
$$

where Re denotes the small-scale Reynolds number defined using the driving flow. The fastest growing mode is found to be fully helical.

This simple model indicates that some driving flows, not satisfying the hypotheses of the $A K A$-effect, described in [11], can generate a negative eddy-viscosity instability satisfying $\sigma \propto q^{2}$. The largest growth-rate is obtained for $\lambda=0$ while no $q^{2}$ instability is predicted for $\lambda=1$. For $\lambda \neq 1$ the flow becomes unstable when the $\beta$ term can overcome the viscosity $\beta>\nu$. This happens when $R e$ is above a critical value: $R e^{c}=b^{-1 / 2}$.

\section{RESULTS}

\section{A. $A K A$}

We begin by examining a flow that satisfies the conditions for an $A K A$ instability. Such a flow was proposed in [11] (from now on Fr87) and is given by:

$$
\begin{aligned}
& U_{x}^{F r 87}=U_{0} \cos \left(K y+\nu K^{2} t\right), \\
& U_{y}^{F r 87}=U_{0} \sin \left(K x-\nu K^{2} t\right), \\
& U_{z}^{F r 87}=U_{x}^{F r 87}+U_{y}^{F r 87} .
\end{aligned}
$$

The growth-rate of large-scale unstable modes can be calculated in the small Reynolds number limit and is given by:

$$
\sigma=\alpha q-\nu q^{2}
$$

with $\alpha=a \operatorname{Re} U_{0}$ and $a=\frac{1}{2}$. The fastest growing mode has negative helicity and $\boldsymbol{q}$ along the $z$-direction.

Setting $\boldsymbol{q}$ along the $z$-direction, we integrated eq. (9)) numerically and measured the growth-rate $\sigma$. Fig. 3 displays the growth-rate of the most unstable mode as a function of the wavenumber amplitude $q=|\boldsymbol{q}|$ for three different values of $R e$ measured by the Floquet code and compared to the theoretical prediction. The agreement is good for small values of $q$ and for small values of $R e$ where the asymptotic limit is valid. For $q$ small enough, the flow is unstable and satisfies $\sigma \propto q$. Fig. 4 shows in log-log scale the growth-rate of the perturbation as a function of $q$ for different Reynolds numbers. The solid line in the graph indicates the $\sigma \propto q$ scaling which is satisfied for all Re. In fig. 5. we compare the theoretical and numerically calculated prefactor $a$ of the $\alpha$ coefficient. This coefficient increases linearly with $R e$ and is seen to be in good agreement with the theoretical prediction up to $R e \simeq 10$. For larger values of $R e, a$ deviates from the linear prediction and saturates. 


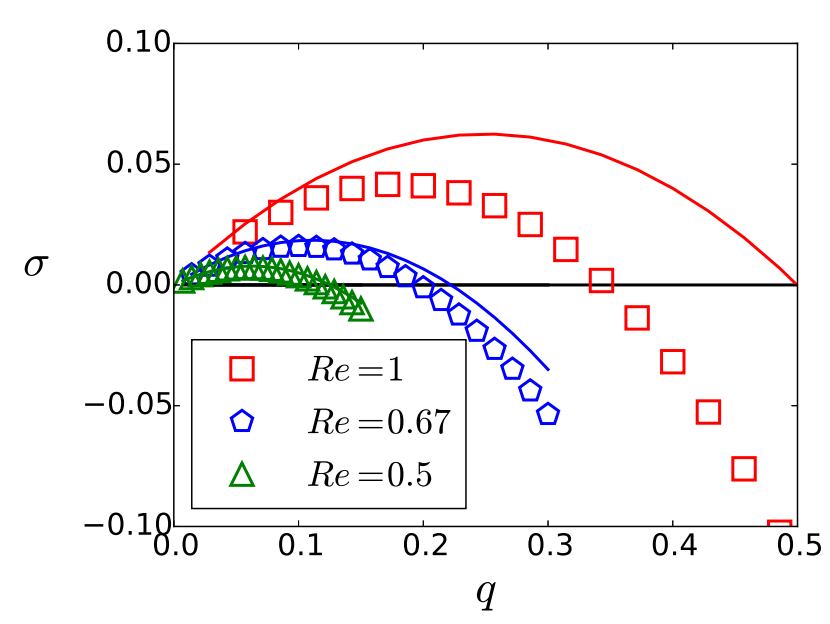

FIG. 3: Growth-rate vs. Floquet wavenumber, $\sigma(q)$, at different Re plotted in log-log scale for a Fr87flow, eq. (24).

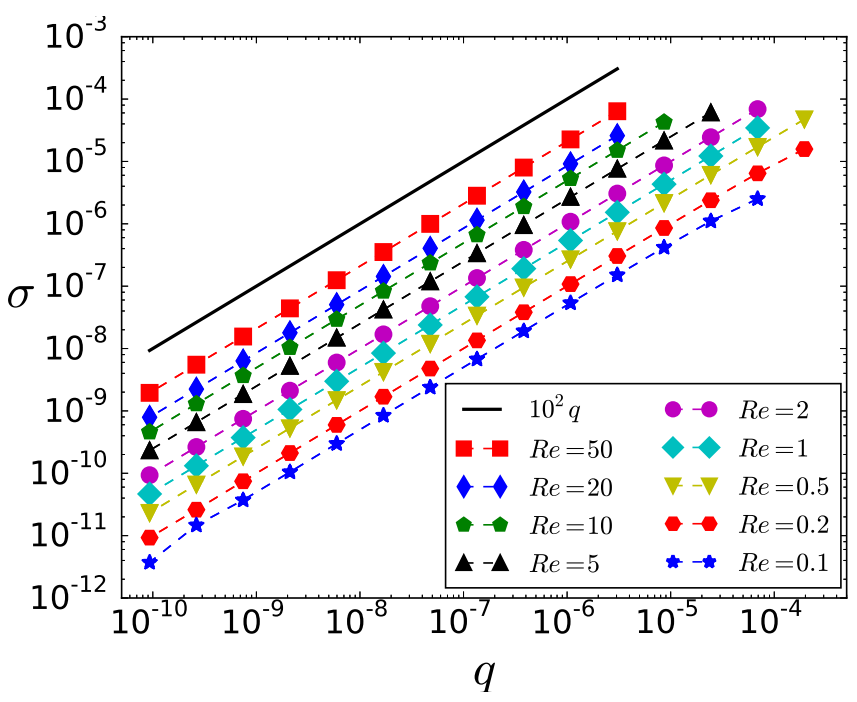

FIG. 4: Growth-rate vs. Floquet wavenumber, $\sigma(q)$, at different $R e$ plotted in log-log scale for a $F r 87$ flow, eq. (24).

A positive growth-rate for a small $q$ mode does not guarantee the dominance of large scales. We should also consider what fraction of the perturbation energy is concentrated in the large scales. Fig. [6 shows the energy spectra for different Reynolds numbers. The energy spectrum for the complex Floquet field $\tilde{\boldsymbol{v}}$ is defined as: $E(k)=\sum_{k-\frac{1}{2} \leq|\mathbf{k}| \leq k+\frac{1}{2}}|\tilde{\boldsymbol{v}}|^{2}$ with $E(k=0)$ the energy at large scales $1 / q$. While at small Reynolds numbers, the smallest wavenumber $k=0$ dominates, as the Reynolds number increases, more energy is concentrated in the wavenumber of the driving flow $k=1$.

To quantify this behavior, we plot in fig. 7 the fraction of the energy in the zero mode $E_{0}=E(0)$ divided by the total energy of the perturbation $E_{t o t}=\sum_{k=0}^{\infty} E(k)$, as a function of the wavenumber $q$ for different values of $R e$. In the small $q$ limit, this ratio reaches an asymptote that depends on the Reynolds number. This asymptotic value

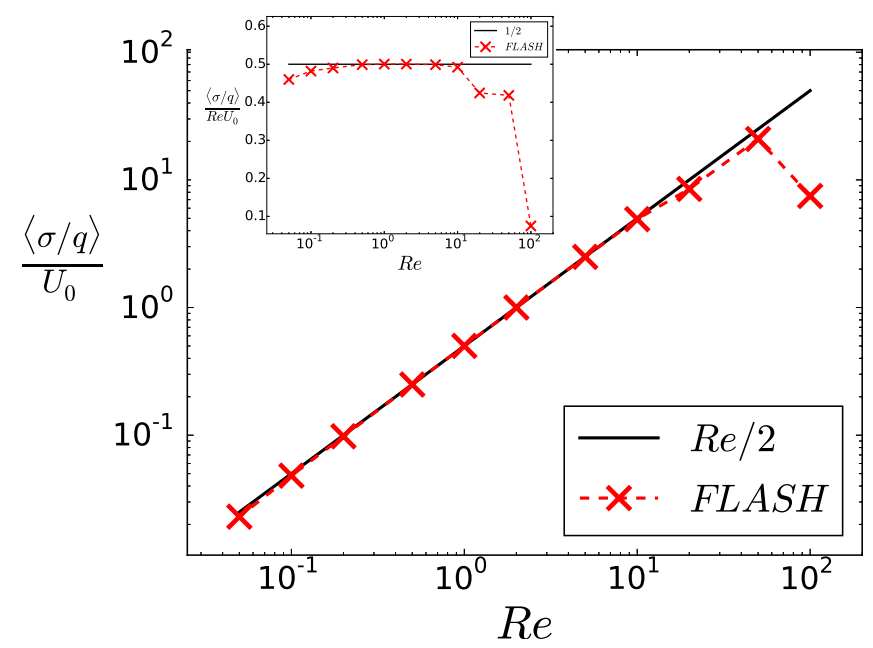

FIG. 5: $\frac{\langle\sigma / q\rangle}{U_{0}}$ coefficient vs. Reynolds number, plotted in $\log$ - log scale for an instability generated by a Fr87 flow, eq. (24). In insert $\frac{\langle\sigma / q\rangle}{R e U_{0}}$ vs. Reynolds number plotted in lin-log scale.

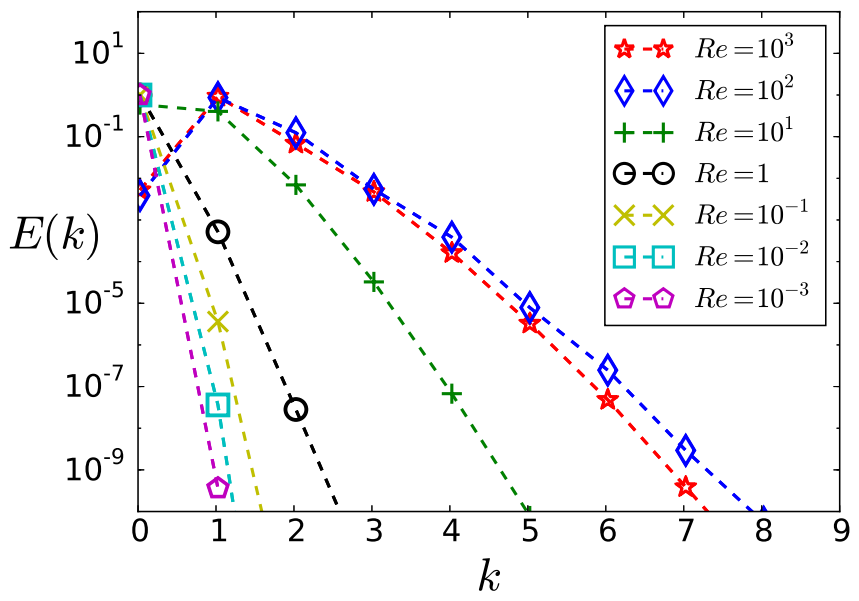

FIG. 6: Spectrum of the Floquet perturbation, $E(k)$, for different small-scale Reynolds numbers, $R e$, with $\boldsymbol{q}=(0 ; 0 ; 0.025)$ generated by a Fr87 flow, eq. (24).

is shown as a function of the $R e$ in fig. 8. The small-scale energy $\left(E_{t o t}-E_{0}\right)$ is then shown to follow a power law $1-\frac{E_{0}}{E_{t o t}} \propto R e^{2}$ for small values of $R e$. Therefore, for the $A K A$ instability, at small $R e$, the energy is concentrated in the large scales, whereas, at large $R e$, the most unstable mode has a small projection in the large scales.

\section{B. Roberts flow: $\lambda=0$}

We now investigate non- $A K A$-unstable flows. We consider the family of the $A B C$ flow, for which we expect large-scale instabilities of the form given in eq. (23). The three-modes model predicts that from the family of $A B C$ flows the most unstable is the $A=1: B=1: C=0$ flow 


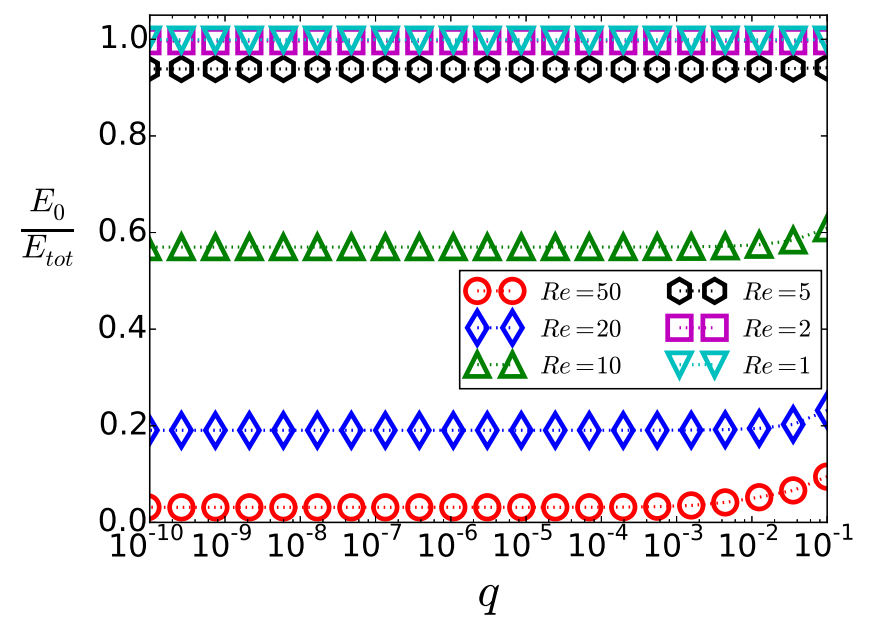

FIG. 7: Growth-rate vs. Floquet wavenumber, $\sigma(q)$, at different Re plotted in log-log scale for a Fr87 flow, eq. (24).

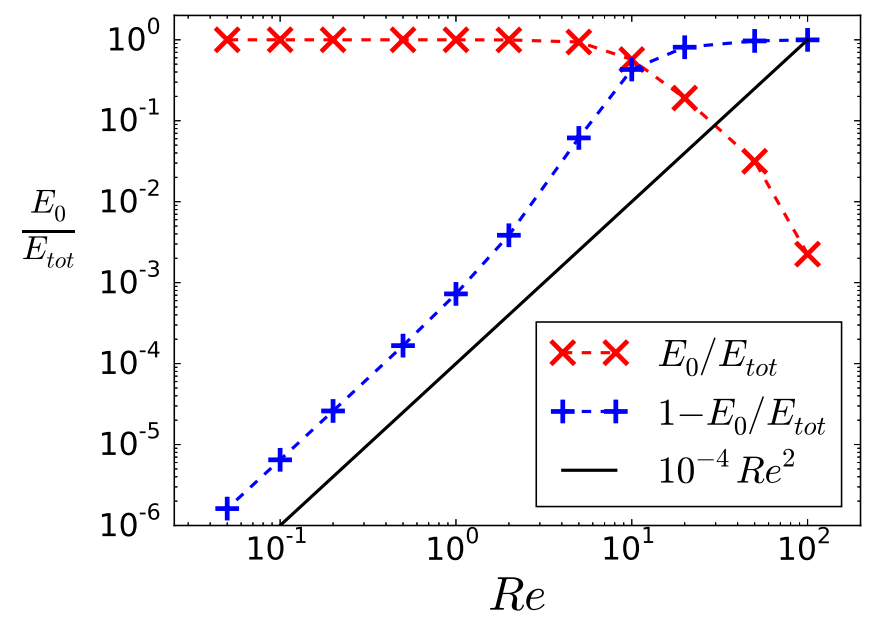

FIG. 8: Energy ratio vs. Reynolds number, $E_{0}$, plotted in log-log scale for a Fr87 flow, eq. (24).

that is commonly referred to as the Roberts flow in the literature [19]. The model predicts a positive growthrate when $R e>2$. Fig. 9 shows the growth-rate $\sigma$ as a function of $q$ for various Reynolds numbers calculated using the Floquet code. For small values of the Reynolds number all modes $q$ have negative growth-rate. Above a critical value $R e^{c} \simeq 2$ unstable modes appear at small values of $q$ in agreement with the model predictions.

To investigate the behavior of the instability for small values of $q$ we plot in Fig. 10 the absolute value of the growth-rate as a function of $q$, in a logarithmic scale, for Reynolds number ranging from 0.312 to 160 . Dashed lines indicate positive growth-rates while dotted lines indicate negative growth-rates. The solid black line indicates the $\sigma \propto q^{2}$ scaling followed by all curves. Therefore, the scaling predicted by the model (eq. (22), (223) is verified. We will refer to the instabilities that follow this scaling $\sigma \propto q^{2}$ as negative eddy-viscosity instabilities.

To further test the model predictions we measure the

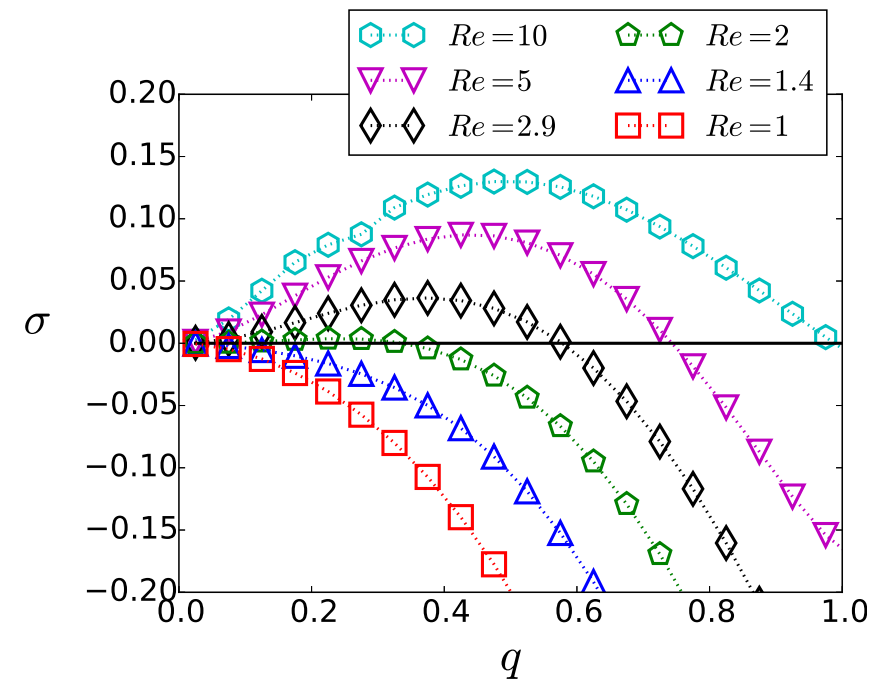

FIG. 9: Growth-rate $\sigma$ vs. Floquet wavenumber $q$, for different $R e$ for the Roberts flow.

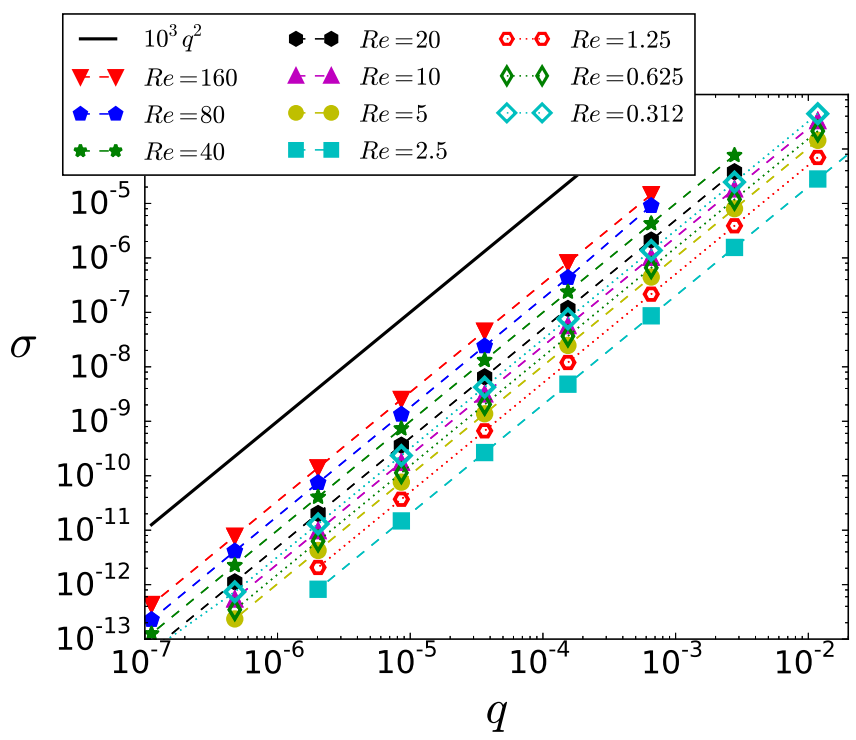

FIG. 10: Growth-rate vs. Floquet wavenumber, $\sigma(q)$, for different $R e$ plotted in log-log scale for a Roberts flow. The

full markers with dashes represent the value of positive growth-rates whereas the empty markers with dots represent the absolute value of negative growth-rates.

proportionality coefficient for the $q^{2}$ power law obtained from the Floquet code. Fig. 11] compares the $b$ coefficient predicted by the three-modes model with the results of the Floquet code. The figure shows $\left(\left\langle\sigma / q^{2}\right\rangle+\nu\right) / \nu$ measured from the data for different values of $R e$, while the $R e^{2} / 4$ prediction of the model is shown by a solid black line. The two calculations agree on nearly two orders of magnitude. Positive growth-rate for the large-scale modes implies $\frac{\left\langle\sigma / q^{2}\right\rangle+\nu}{\nu}>1$. The critical value of the Reynolds number, for which the instability begins, can be obtained graphically at the intersection of the numer- 
ically obtained curve with the $\frac{\left\langle\sigma / q^{2}\right\rangle+\nu}{\nu}=1$ line plotted with a dash-dot green line. The predictions of the model $R e^{c}=2$ and the numerically values obtained are in excellent agreement.

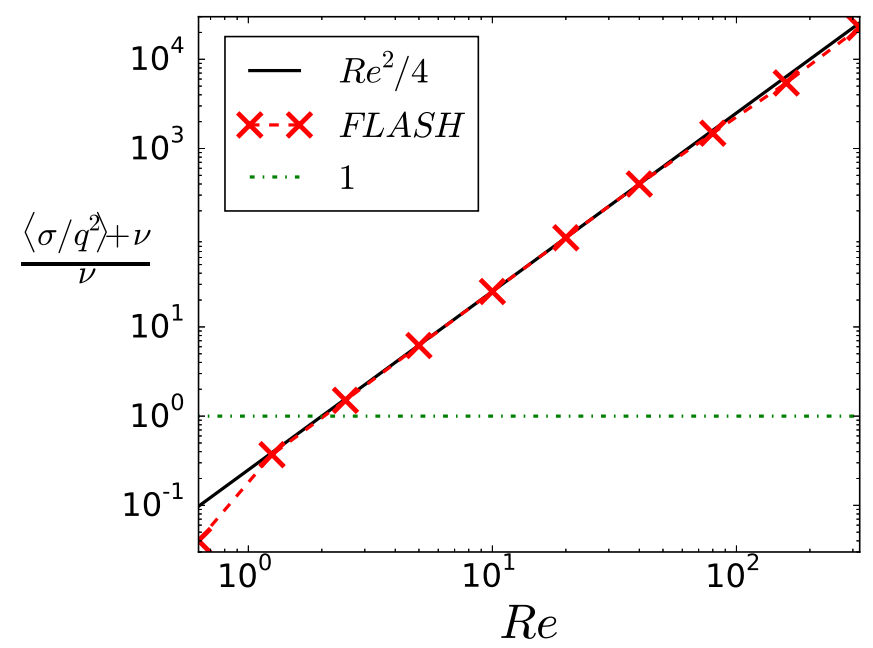

FIG. 11: $\frac{\left\langle\sigma / q^{2}\right\rangle+\nu}{\nu}$ vs. of Reynolds number, plotted in lin-log scale for the Roberts flow.

Similarly to the $A K A$ flow, the fraction of energy concentrated in the large scales $(k=1)$ becomes independent of $q$ in the small $q$ limit. This is demonstrated in fig. 12 where the ratio of $E_{0} / E_{t o t}$ is plotted as a function of $q$. In fig. 13, we show the asymptotic value of this ratio as a function of the Reynolds number. As in the case of the $A K A$ instability, the projection to the large scales depends on the Reynolds number, and at large $R e$, it follows the power law $\frac{E_{0}}{E_{t o t}} \propto R e^{-2}$.

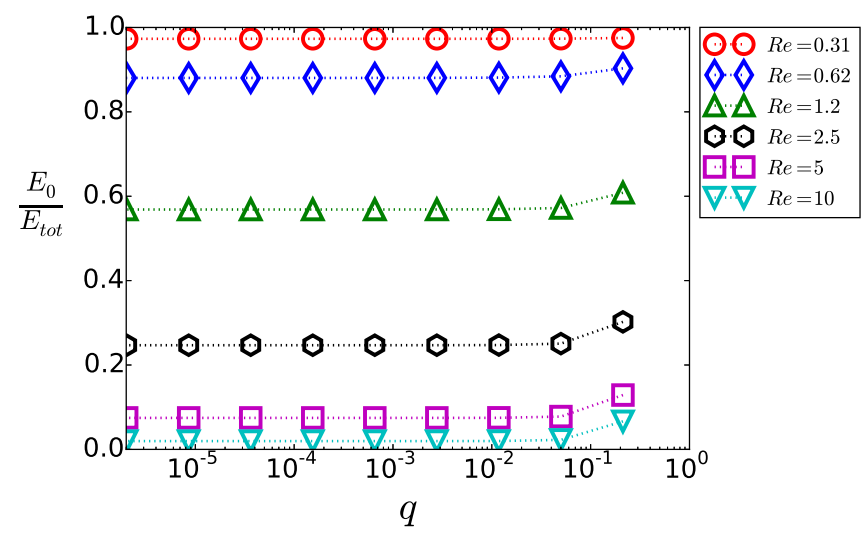

FIG. 12: Growth-rate vs. Floquet wavenumber, $\sigma(q)$, at different $R e$ plotted in log-log scale for a Roberts flow.

\section{Equilateral $A B C$ flow: $\lambda=1$}

For the $A=1: B=1: C=1$ flow, the three-modes model predicts that the $b$ coefficient is zero. Therefore,

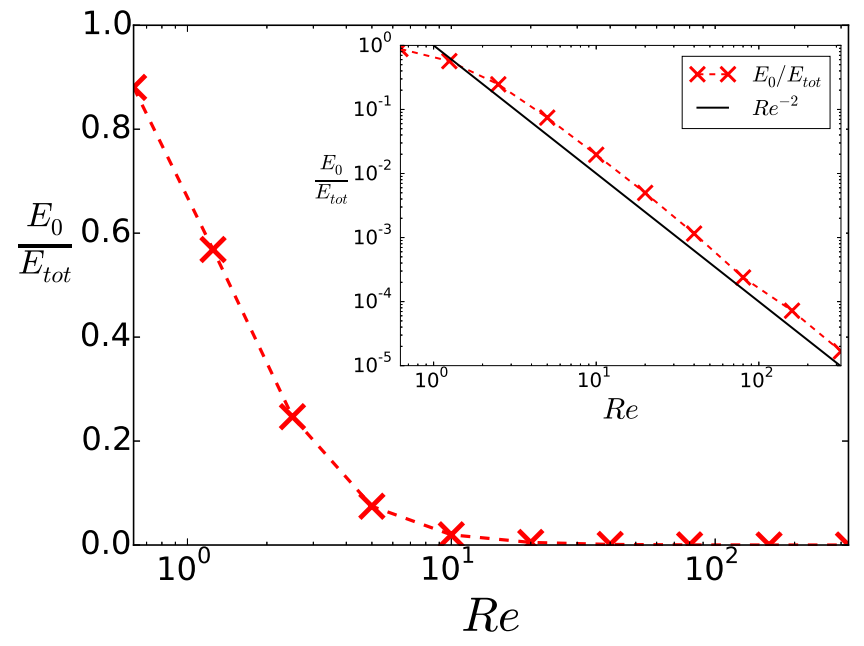

FIG. 13: Fraction of large-scale energy, $\frac{E_{0}}{E_{t o t}}$, for different Reynolds number for the most unstable mode of the Roberts flow.

the model does not predict a negative eddy-viscosity instability with: $\sigma \propto q^{2}$. Fig. 14 shows the growth-rate as a function of the wavenumber $q$ calculated using the Floquet code for different values of the Reynolds number. Clearly the small $q$ modes still become unstable but the dependence on $R e$ appears different from the previously examined cases. We thus examine separately the small Re and large Re behaviors.

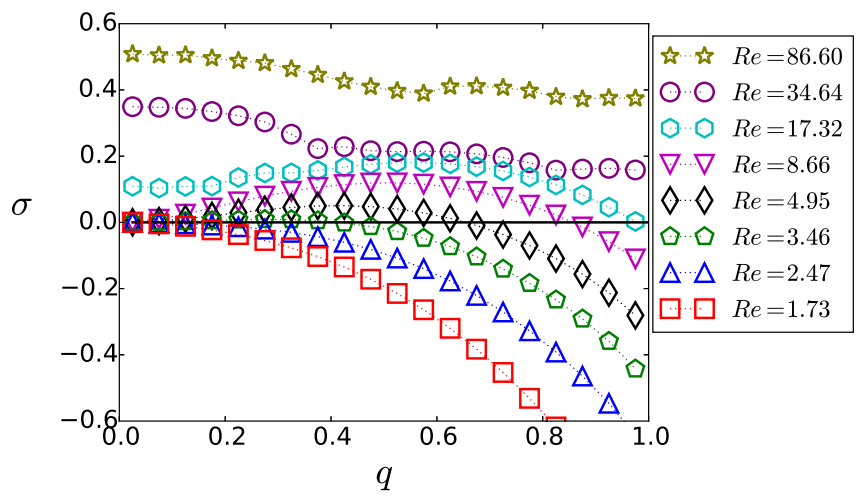

FIG. 14: Growth-rate vs. Floquet wavenumber $\sigma(q)$, for different $R e$ for the $A B C$ flow.

\section{Small values of Re}

First, we examine the instability for small values of $R e \leq 10$ for which the growth-rate $\sigma$ tends to zero as $q \rightarrow 0$. Fig. 15] shows the growth-rate of the instability for the equilateral $A B C$ flow as a function of the wavenumber $q$ in logarithmic scale for different values of $R e$ ranging from 0.312 to 10 . In this range, the growth-rate behaves much like the Roberts flow, and is in contradiction with the three-modes model. The numerically calculated 
growth-rates show a clear negative eddy-viscosity scaling $\sigma \propto q^{2}$. The growth-rate becomes positive above a critical value of $R e$.

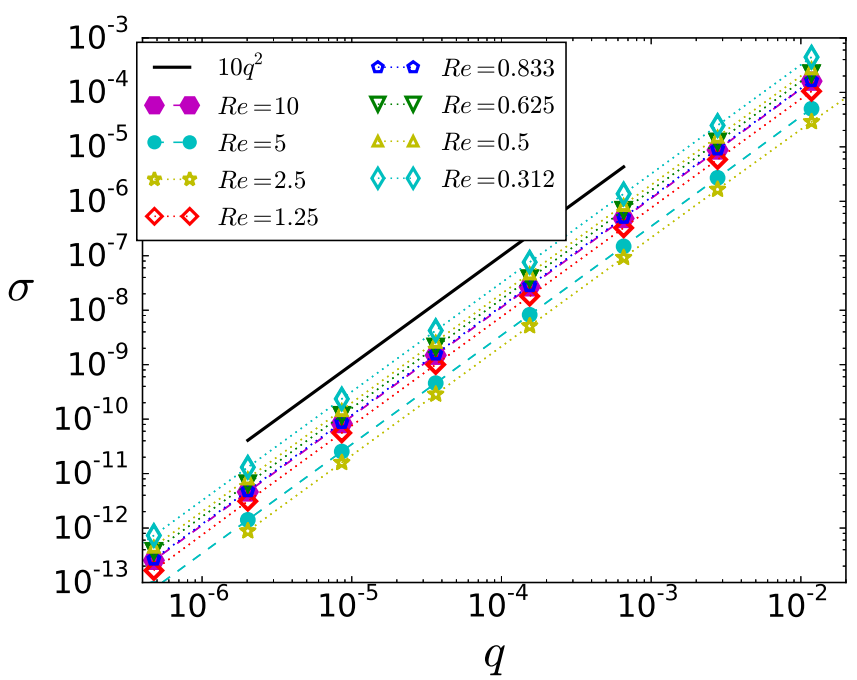

FIG. 15: Growth-rate vs. Floquet wavenumber, $\sigma(q)$, for different $R e$ plotted in log-log scale for a equilateral $A B C$ flow. The full markers with dashes represent the value of positive growth-rates whereas the empty markers with dots represent the absolute value of negative growth-rates.

In fig. 16, the measured value of $\frac{\left\langle\sigma / q^{2}\right\rangle+\nu}{\nu}$ is represented as a function of the Reynolds number. In the insert, the plot lin-log of $\frac{\left\langle\sigma / q^{2}\right\rangle+\nu}{R e^{2} \nu}$ provides a measurement of the $b$ coefficient. This expression becomes larger than one (signifying the instability boundary that is marked by a dash-dot line) for $R e \gtrsim 3$. This value $R e^{c} \simeq 3$ is slightly higher than the critical Reynolds number of the Roberts flow $R e^{c}=2$. At very small Reynolds number, the value of $b=\frac{\left\langle\sigma / q^{2}\right\rangle+\nu}{R e^{2} \nu}$ approaches zero very quickly, which indicates that the model prediction is recovered at $R e \rightarrow 0$.

To investigate further the discrepancy of the Floquet results with the three-modes model. Fig. 17 shows the $b$ coefficient (measured as $b=\frac{\left\langle\sigma / q^{2}\right\rangle+\nu}{R e^{2} \nu}$ ) for different $\lambda$ parameter from 0 (Roberts flow) to 1 (equilateral $A B C$ flow). All the DNS are carried out at $R e=10$. The results indicate that the three-modes model and the results from the Floquet code agree for $\lambda \lesssim 0.5$ but deviate as $\lambda$ becomes larger. To identify where this discrepancy between the model and the DNS occurs, we modified the FLASH code in order to test the assumptions of the model. This is achieved by enforcing the adiabatic approximation in the Floquet code and by controlling the number of modes that play a dynamical role. The later is performed by using a Fourier truncation of the Floquet perturbation at a value $k_{c u t}$ so that only modes with $k<k_{\text {cut }}$ are present. Fig 18 shows the dependence of the $b$ coefficient on the truncation mode, $k_{c u t}$. For $k_{c u t} \geq 3$, the growth-rate reaches the asymptotic value that is also observed in the insert of fig. 16 for $R e=10$

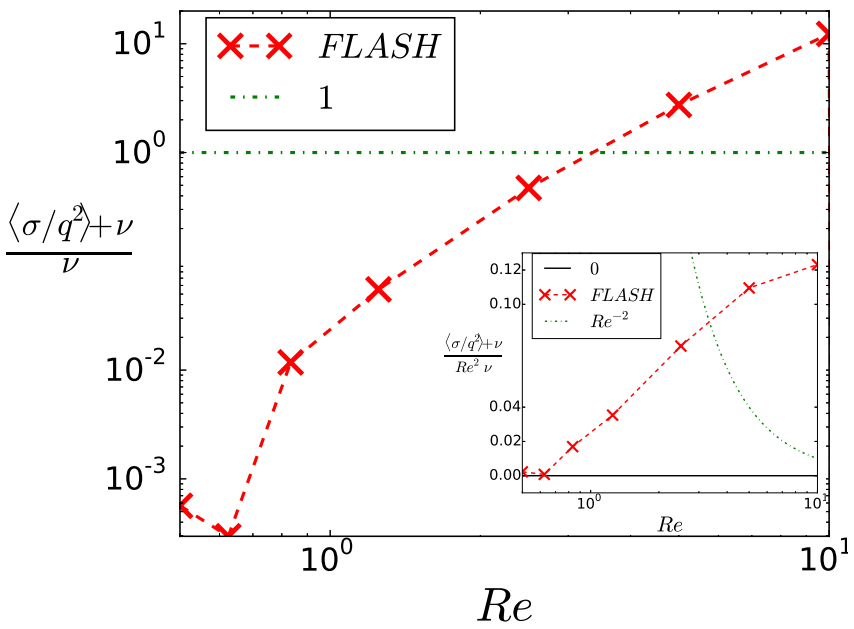

FIG. 16: $\frac{\left\langle\sigma / q^{2}\right\rangle+\nu}{\nu}$ coefficient vs. Reynolds number, plotted in $\log -\log$ scale for the equilateral $A B C$ flow. The insert shows $b=\frac{\left\langle\sigma / q^{2}\right\rangle+\nu}{R e^{2} \nu}$.

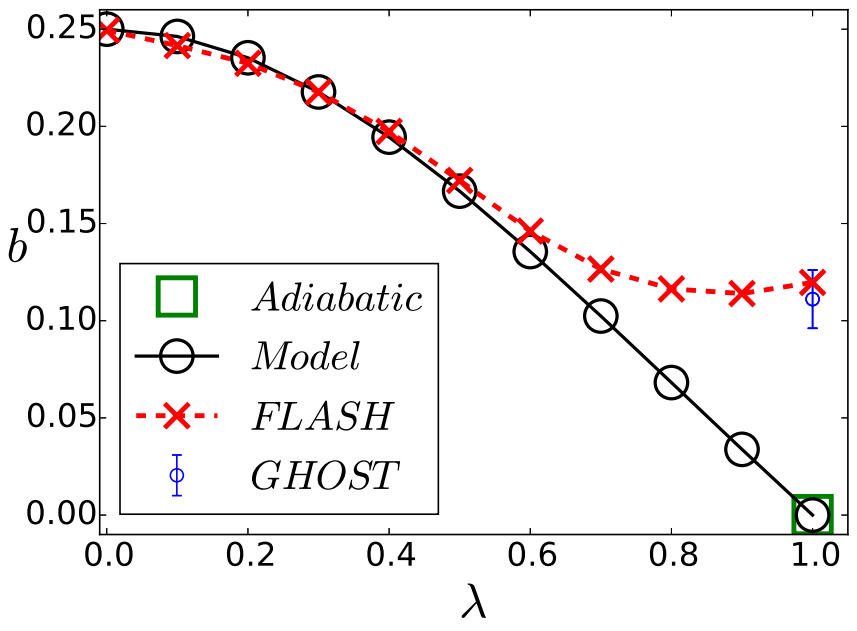

FIG. 17: $b$ coefficient vs. $\lambda$ parameter, $b(\lambda)$, at $R e=10$ for $\lambda-A B C$ flows with parameter: $A=1: B=1: C=\lambda$.

obtained from the "untampered" FLASH code. This confirms the assumption that modes in the smallest scales have little impact on the evolution of the large-scale perturbation. However, the $b$ coefficient strongly varies for $k_{\text {cut }} \leq 3$. The model predictions are recovered only when $k_{c u t}=1$ that amounts to keeping only the modes used in the model. Therefore, the hypothesis of the model to restrict the interaction of the perturbation to its first two Fourier modes does not seem to hold for the equilateral $A B C$ flow at moderate Reynolds number, $1 \leq R e \leq 10$. The adiabatic hypothesis does not appear to affect the results. Therefore, the discrepancy between the threemodes model and the numeric results is due to the coupling of the truncated velocity $\boldsymbol{v}_{>}$that was neglected in the model. 


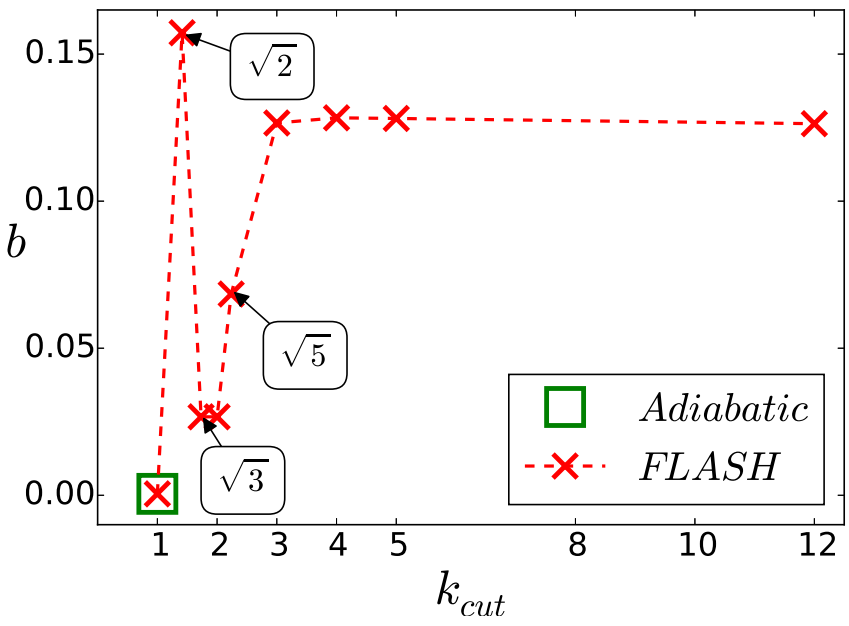

FIG. 18: $b$ coefficient vs. Fourier truncation mode, $b\left(k_{c u t}\right)$, at $R e=10$ of instabilities generated by $\lambda-A B C$ flows.

\section{Large values of Re}

We now turn our focus to large values of the Reynolds number that display a finite growth-rate $\sigma$ at $q \rightarrow 0$, see fig. 14. Fig. 19] shows the growth-rate $\sigma$ in a lin-log scale for four different values of the Reynolds number. Unlike the small values of $R e$ examined before here it is clearly demonstrated that above a critical value of $R e$ the growth-rate $\sigma$ reaches an asymptotic value independent of $q$. At first, this finite growth-rate seems to violate the momentum conservation. Indeed, momentum conservation enforces modes with $q=0$, corresponding to uniform flows, not to grow.

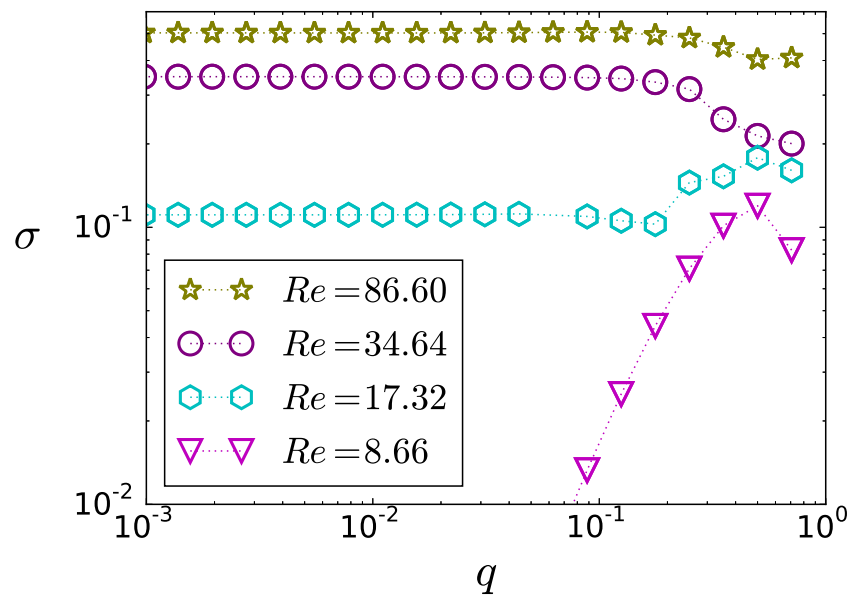

FIG. 19: Growth-rate as a function of $q$ for the $A B C$ flow and for large values of $R e$

The resolution of this conundrum can be obtained by looking at the projection of the unstable modes to the large scales. In fig. 20, we plot the ratio $E_{0} / E_{\text {tot }}$ as a function of $q$ for the same values of $R e$ as used in fig. 19. Unlike the small $R e$ cases examined previously, for large $R e$, this energy ratio decays to zero at small values of $q$ and appears to follow the power law $E_{0} / E_{\text {tot }} \propto q^{4}$. Therefore, at $q=0$, the energy at large scales $E_{0}$ is zero and the momentum conservation is not violated in the $q=0$ limit.

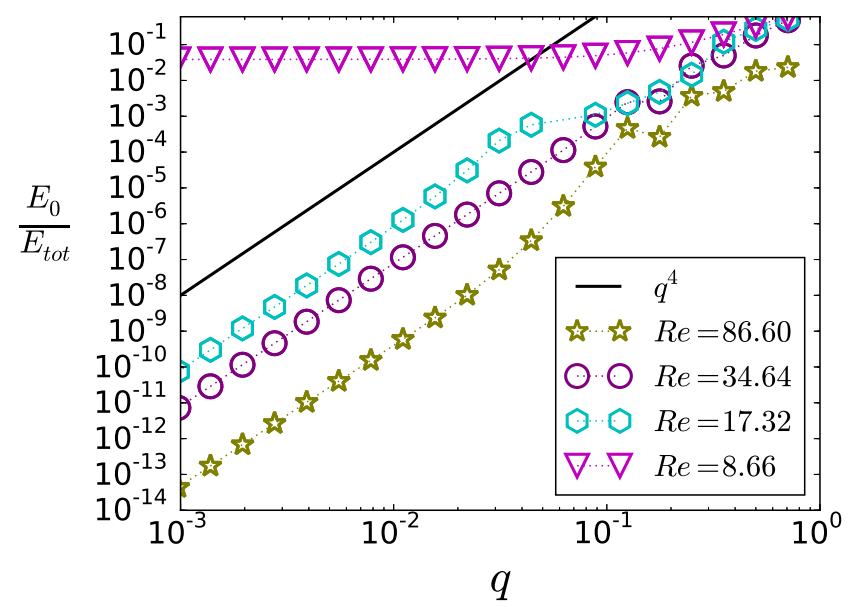

FIG. 20: Growth-rate as a function of $q$ for the $A B C$ flow and for large values of $R e$

\section{Small and large-scale instabilities}

It appears that there are two distinct behaviors: the first one for which $\lim _{q \rightarrow 0} \sigma=0$ and $\lim _{q \rightarrow 0} E_{0} / E_{t o t}>$ 0 when $R e$ is small and the second one for which $\lim _{q \rightarrow 0} \sigma>0$ and $\lim _{q \rightarrow 0} E_{0} / E_{\text {tot }}=0$ when $R e$ is large. We argue that there is a second critical Reynolds number $R e_{S}^{c}$ such that flows for which $R e^{c}<R e<R e_{S}^{c}$ show the first behavior while flows with $R e_{S}^{c}<R e$ show the second behavior. This second critical value is related to the onset of small-scale instabilities.

To demonstrate this claim we are going to use a simple model. We consider the evolution of two modes, one at large scales $v_{q}$ and one at small scales $v_{Q}$. These modes are coupled together by an external field $U$. In the absence of this coupling, the large-scale mode $v_{q}$ decays while the evolution of the small-scale mode $v_{Q}$ depends on the value of the Reynolds number. The simplest model satisfying these constraints, dimensionally correct and leading to an $A K A$ type $\sigma \propto q$ instability or a negative eddy-viscosity instability $\sigma \propto q^{2}$ is:

$$
\begin{aligned}
\frac{d}{d t} v_{q} & =-\nu q^{2} v_{q}+U q^{n} Q^{1-n} v_{Q}, \\
\frac{d}{d t} v_{Q} & =U Q v_{q}+\sigma_{Q} v_{Q} .
\end{aligned}
$$

The index $n$ takes the values $n=1$ if an $A K A$ instability is considered and $n=2$ if an instability of negative eddyviscosity is considered. Note that for $q=0$ the growth of $v_{q}$ is zero, as required by momentum conservation. $\sigma_{Q}=s U Q-\nu Q^{2}$ gives the small-scale instability growthrate that is positive if $R e=U /(\nu Q)>1 / s=R e_{S}^{c}$. 
The simplicity of the model allows for an analytical calculation of the growth-rate and the eigenmodes. Despite its simplicity, it can reproduce most of the results obtained here in the $q \ll Q$ limit. The general expression for the growth-rate is given by $\sigma=$ $\frac{1}{2}\left[\left(\sigma_{Q}-\nu q^{2} \pm \sqrt{\left(\sigma_{Q}+\nu q^{2}\right)^{2}+4 Q^{2-n} q^{n} U^{2}}\right]\right.$ and eigenmode satisfies $v_{q} / v_{Q}=U q^{n} Q^{1-n} /\left(\sigma+\nu q^{2}\right)$.

First, we focus on large values of $\nu$ such that $\sigma_{Q}=$ $-\nu Q^{2}<0$. For $n=1$, the growth-rate $\sigma$ and the energy ratio $E_{0} / E_{t o t}=v_{q}^{2} /\left(v_{q}^{2}+v_{Q}^{2}\right)$ are given to the first order in $q$

$$
\sigma \simeq \frac{U^{2} q}{\nu Q} \quad \text { and } \quad \frac{E_{0}}{E_{t o t}} \simeq \frac{1}{1+R e^{2}} .
$$

In the same limit for $n=2$ we obtain

$$
\sigma \simeq \nu\left(R e^{2}-1\right) q^{2} \text { and } \quad \frac{E_{0}}{E_{t o t}} \simeq \frac{1}{1+R e^{2}} .
$$

The critical Reynolds number for the large-scale instability is given by $R e^{c}=1$. Both of these results in eqs. (28), (29) are in agreement with the results demonstrated in figs. 4, 7, 8, 10, 12, 13,

The behavior changes when a small-scale instability exists $\sigma_{Q}>0$. This occurs when $U Q>s \nu Q^{2}$ at the critical Reynolds number: $\operatorname{Re}_{S}^{c}=1 / s$. For large $R e \gg$ $R e_{S}^{c}$ we thus expect $\sigma_{Q} \simeq s U Q>0$. In this case for $n=1$ to first order in $q$, we have:

$$
\sigma \simeq \sigma_{Q} \quad \text { and } \quad \frac{E_{0}}{E_{t o t}} \simeq \frac{q^{2}}{s^{2} Q^{2}}
$$

while for $n=2$, we obtain:

$$
\sigma \simeq \sigma_{Q} \quad \text { and } \quad \frac{E_{0}}{E_{t o t}} \simeq \frac{q^{4}}{s^{2} Q^{4}}
$$

The model is thus in agreement also with the scalings observed in figs. 19, 20. The transition from one behavior to the other occurs at the onset of small-scale instability $R e_{S}^{c}$. It is thus worth pointing out that the results of the FLASH codes showed that the transition from $\lim _{q \rightarrow 0} \sigma=0$ modes to $\lim _{q \rightarrow 0} \sigma>0$ occurs at the value of $R e$ for which small-scale instability of the $A B C$ flow starts $R e_{S}^{c} \simeq 13$ [20]. This further verifies that the transition observed is due to the development of smallscale instabilities.

We also note here that both the Roberts flow and the Fr87 flow given in eq. (24) are invariant in translations along the $z$-direction. This implies that each $q_{z}$ mode evolves independently with out coupling to other $k_{z}$ modes. The onset of small-scale instabilities $R e_{S}^{c}$ for $q=0$ in this case then corresponds to the onset of two dimensional instabilities. Two dimensional flows however forced at the largest scale of the system are known to be stable at all Reynolds numbers [21]. This result originates from the fact that two dimensional flows conserve both energy and enstrophy and small scales cannot be excited without exciting large scales at the same time.
This is the reason why no $R e_{S}^{c}$ were observed in these flows.

Finally, this model provides a way to distinguish between the presence or absence of the $A K A$ effect for values of Re larger than the critical Reynolds for small-scale instabilities $R e_{S}^{c}$ by looking at the scaling of the energy in the large scales with respect to the scale separation $q / Q$. In the presence of an $A K A$ effect the scaling of eq. (30) is expected, while, in the absence of an $A K A$ effect, the scaling of eq. (31) is expected if a negative eddy-viscosity is present.

\section{Turbulent equilateral $A B C$ flows}

As discussed in the introduction the driving flow does not need to be laminar to use Floquet theory. It is only required to obey the $2 \pi \ell$-periodicity. It is worth thus considering large-scale instabilities in a turbulent $A B C$ flow that satisfies the forcing periodicity. This amounts to the turbulent flow forced by an $A B C$ forcing in a periodic cube of the size of the forcing period $2 \pi \ell$. Due to the stationarity of the laminar $A B C$ flow, it can be excluded as possible candidate for an $A K A$ instability. However, this is not true of a turbulent $A B C$ flow since it evolves in time. We cannot thus a priori infer that a turbulent $A B C$ flow results in an $A K A$ instability or not.

To test this possibility, we consider the linear evolution of the large-scale perturbations $\boldsymbol{v}$ driven by an equilateral $A B C$ flow at $R e=50$, that is beyond the onset of the small-scale instability $R e_{S}^{c} \simeq 13$. The turbulent equilateral $A B C$ flow $\mathbf{U}$ is obtained solving the NavierStokes eqs. (3) in the domain $(2 \pi \ell)^{3}$ driven by the forcing function $\mathbf{F} A B C=\mathbf{U}^{A B C}$. The code is executed until the flow reaches saturation. The evolution of the large scale perturbations is then examined solving eq. (9) with the FLASH code coupled to the Navier-Stokes eqs. (3).

The kinetic energy $E_{U}$ of the turbulent equilateral $A B C$ flow $\mathbf{U}$ is shown in fig. 21. The energy $E_{U}$ strongly fluctuates around a mean value. The evolution of the energy $E_{t o t}$ of the perturbations $\mathbf{v}$ for different values of $q$ is shown in the insert of 22, $E_{t o t}$ shows an exponential increase, from which the growth-rate can be measured. The growth-rate $\sigma$ as a function of the wavenumber $q$ is shown in fig. 22 while the ratio $E_{0} / E_{t o t}$ is shown in fig. 23. The growth-rate of the large-scale instabilities appears to reach an finite value in the limit $q \rightarrow 0$ just like laminar $A B C$ flows above the small-scale critical Reynolds $R e_{S}^{c}$. However, the ratio $E_{0} / E_{t o t}$ does not scale like $q^{4}$ as laminar equilateral $A B C$ flows but like $q^{2}$. As discussed in the previous section, this indicates that the turbulent equilateral $A B C$ flow is $A K A$-unstable. This can have possible implications for the saturated stage of the instability that we examine next. 


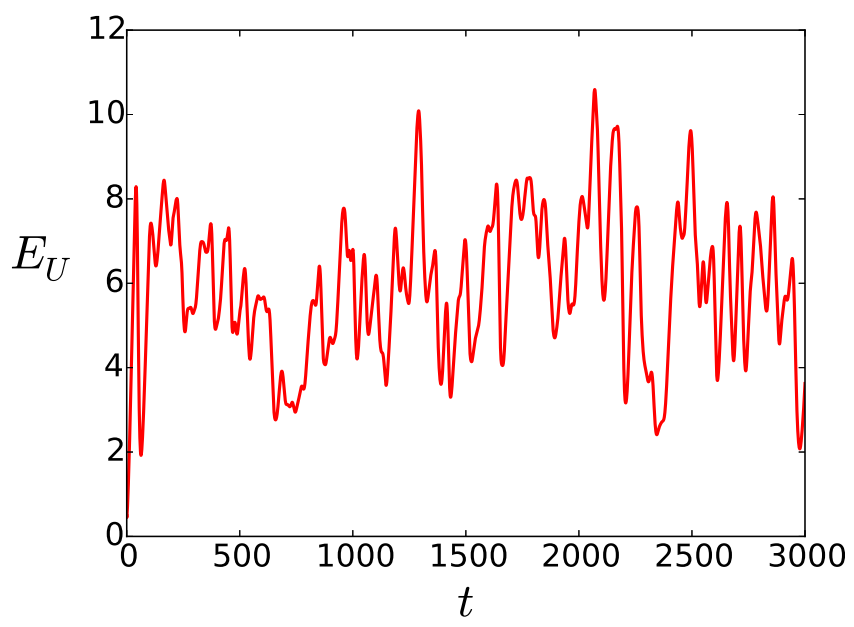

FIG. 21: Energy evolution of the turbulent equilateral $A B C$ driving flow at $R e=122$.

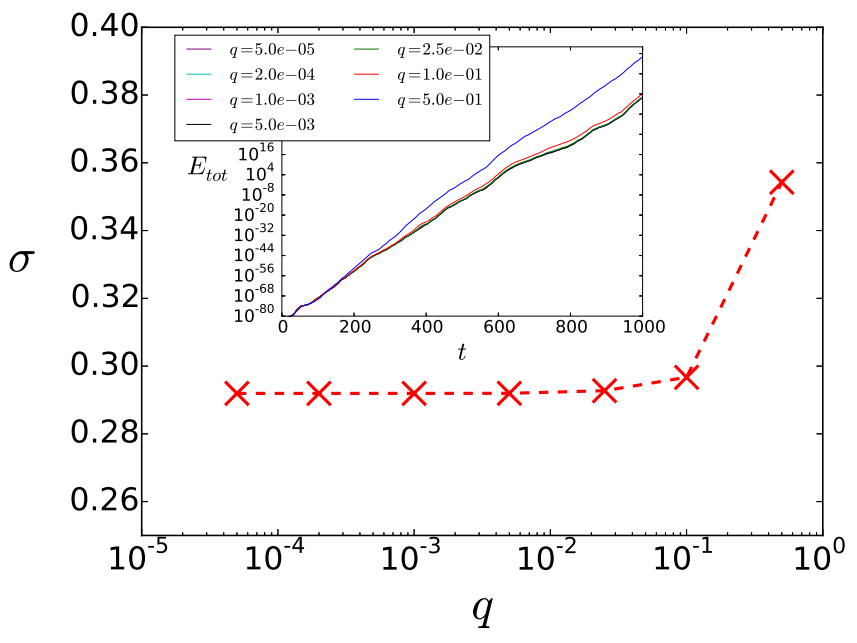

FIG. 22: Growth-rate of the turbulent equilateral $A B C$ driving flow $\mathrm{v}$, the wavenumber, $\sigma(q)$. The insert shows the exponential growth of the large-scale perturbations for various $q$.

\section{E. Non-linear calculations and bifurcation diagram}

We further pursue our investigation of large-scale instabilities by examining the non-linear behavior of the flow close to the instability onset. We restrict ourselves to the case of the equilateral $A B C$ flow whose non-linear behavior has been extensively studied in the absence however of scale separation [18]. The linear stability of the $A B C$ flow in the minimum domain size has been studied in [20] and more recently in 22]. These studies have shown that the $A B C$ flow destabilizes at $R e_{S}^{c} \simeq 13$.

To investigate the non-linear behavior of the flow in the presence of scale separation, we perform a series of DNS of the forced Navier-Stokes equation (eq. (1)) in triple periodic cubic boxes of size $2 \pi L$. The forcing maintaining the flow is $\mathbf{F}^{A B C}=\frac{\sqrt{2}}{\sqrt{3}} \nu|\mathbf{K}|^{2} \mathbf{U}^{A B C}$ so that the laminar

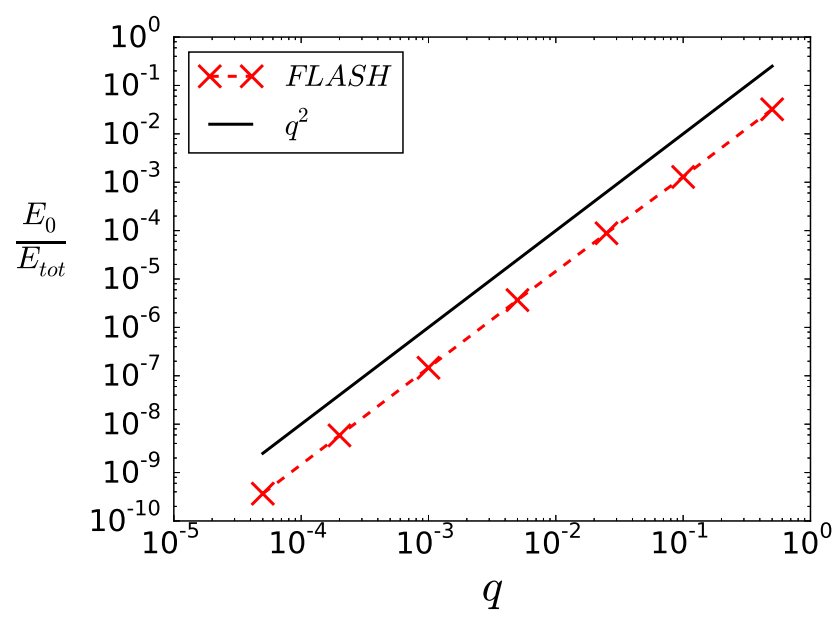

FIG. 23: $E_{0} / E_{\text {tot }}$ ratio vs. the wavenumber $q$.

solution of the flow is the $A B C$ flow [18] normalized to have unit energy. Four different boxes sizes are considered: $K L=1,5,10$ and 20. For each box size and for each value of $R e$, the flow is initialized with random initial conditions and evolves until a steady state is reached.

Fig. 24 shows the saturation level of the total energy $E_{V}$ at steady state as a function of $R e$ for the four different values of $K L$. At low Reynolds number, the laminar solution $\mathbf{V}=\mathbf{U}^{A B C}$ is the only attractor and so the energy is $E_{V}=1$. At the onset of the instability the total energy decreases. A striking difference appears between the $K L=1$ case and other three cases. For the $K L=1$ case the first instability appears at $R e_{S}^{c} \simeq 13$ in agreement with the previous work [20, 22]. By definition, only small-scale instabilities are present in the $K L=1$ case (i.e. instabilities that do not break the forcing periodicity). For the other three cases, which allow the presence of modes of larger scale than the forcing scale, the flow becomes unstable at a much smaller value: $R e^{c} \simeq 3$. This value of $R e^{c}$ is in agreement with the results obtained in section $\Pi$ for large-scale instability by a negative eddyviscosity mechanism. The energy curves for the forcing modes $K L \geq 5$ all collapse on the same curve. This indicates that not only the growth-rate but also the saturation mechanism for these three simulations are similar.

Further insight on the saturation mechanism can be obtained by looking at the energy spectra. Fig. 25] shows the energy spectrum of the velocity field at the steady state of the simulations. Two types of spectra are plotted. In fig. 25, spectra plotted using lines and denoted as $k$-bin display energy spectrum collected in bins where modes $\mathbf{k}$ satisfy $n_{1}-1 / 2<|\mathbf{k}| L \leq n_{1}+1 / 2$, with $n_{1}$ a positive integer. $E(k)$ then represents the energy in the bin $n_{1}=k$. In fig. 25, spectra plotted using red dots and denoted by $k^{2}$-bin display the energy spectrum collected in bins where modes $\mathbf{k}$ satisfy $|\mathbf{k}|^{2} L^{2}=n_{2}$, with $n_{2}$ a positive integer. Since $\mathbf{k} L$ is a vector with integer components $m_{x}, m_{y}$ and $m_{z}$, its norm $k^{2} L^{2}=m_{x}^{2}+m_{y}^{2}+m_{z}^{2}$ is also a positive integer. $E(k)$ then represents the energy in 


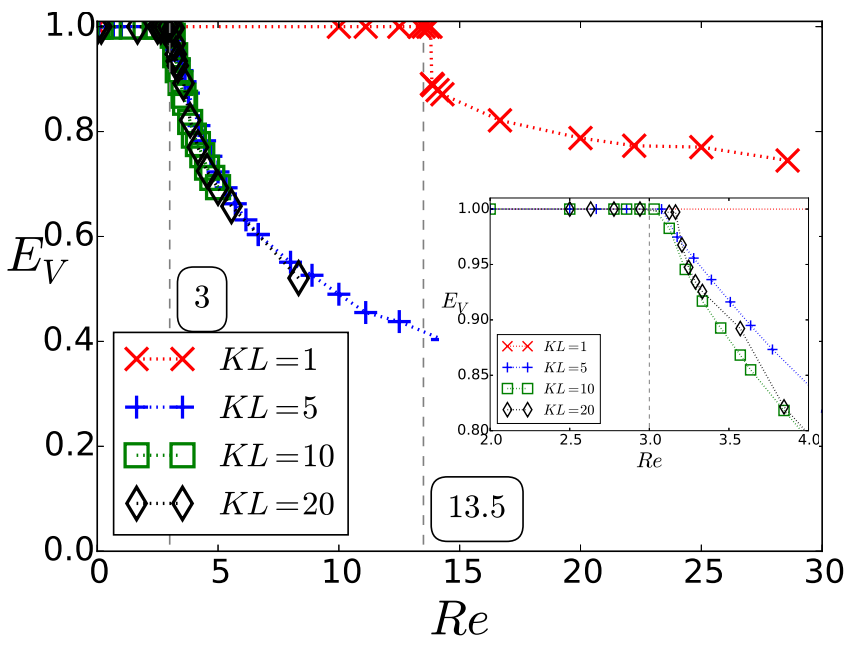

FIG. 24: Bifurcation: total energy vs. Reynolds number, $E_{\text {tot }}(R e)$, for different scale separation $K \in\{1 ; 5 ; 10 ; 20\}$. In insert, zoom of the graph for $R e \in[2 ; 5]$.

the bin $n_{2}=k^{2} L^{2}$. This type of spectrum provides more precise information about the energy distribution among modes. In our case, they help separate $K$ modes from $K \pm 1 / L$ modes and highlight the three-modes interaction. The $k=K \pm 1 / L$ modes as well as the largest scale mode $k L=1$ that were used in the three-modes model are shown by blue circles in the spectra. The drawback of $k^{2}$-bin spectra is their memory consumption. They have a number of bins equal to the square of the number of bins of standard $k$-bin spectra. However, since spectra are not outputted at every time-step, this inconvenience is limited.
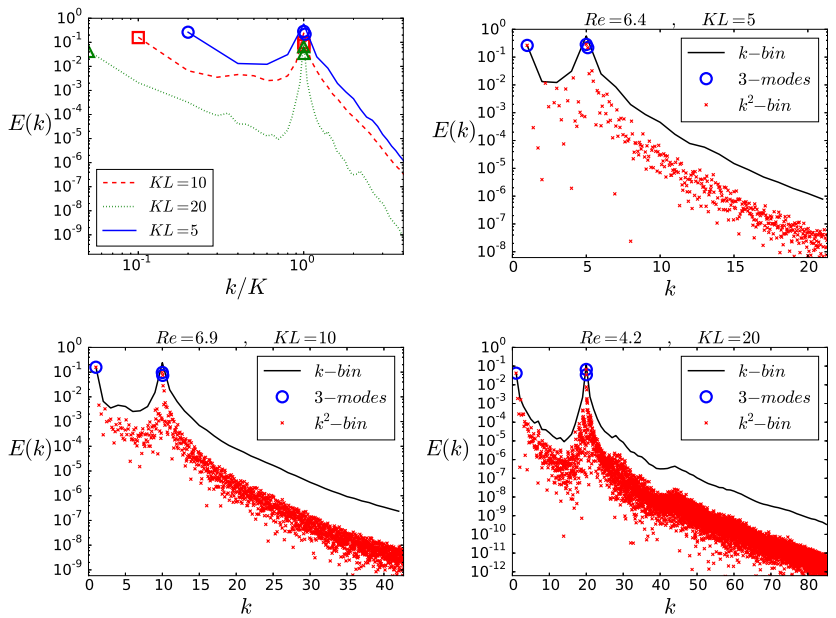

FIG. 25: Energy spectra, $E(k)$, for different scale separation $K \in\{1 ; 5 ; 10 ; 20\}$.

The plots of the spectra show that the most energetic modes are the modes close to the forcing scale and the largest scale mode $k L=1$. This is true even for the largest scale separation examined $K L=20$. We note that the largest scale mode is not the most unstable one as seen in all the cases examined (see figs. 3[9]14). Despite this fact, it appears that the $k L=1$ is the dominant mode that controls saturation. The exact saturation mechanism however is beyond the scope of this work.

\section{CONCLUSION}

In this work, we examined in detail the large-scale hydrodynamic instabilities of a variety of flows. Using the Floquet framework as well as simplified models, we were able to investigate the stability of periodic flows to large-scale perturbations for a wide parameter range. Our work verifies the asymptotic results derived in the past but also covers cases that go beyond their validity including turbulent flows.

For the Fr87 flow (see eq. (24)) at small values of Re, the instability growth rate scales like: $\sigma \propto q R e$, with most of the energy in the large scales $1-E_{0} / E_{\text {tot }} \propto$ $R e^{2}$. It is present for any arbitrarily small value of the Reynolds number provided that scale separation is large enough. When Re becomes of order one this behavior changes. The growth-rate saturates in Re and most of the energy of the most unstable mode is concentrated in the small scales.

Flows in the absence of an $A K A$ effect, like the $A B C$ and Roberts flow, show a negative eddy-viscosity scaling. The instability appears only above a critical value of the Reynolds number $R e^{c}$ that was found to be $R e^{c} \simeq 2$ for the Roberts flow and $R e^{c} \simeq 3$ for the equilateral $A B C$ flow. The growth-rate follows the scaling $\sigma \propto$ $\nu\left(b R e^{2}-1\right) q^{2}$. The value of $b$ can be calculated based on a three mode model for the Roberts flow and was found to be $b=1 / 4$. The three-modes model however failed to predict the $b$ coefficient of the equilateral $A B C$ flow because more modes were contributing to the instability. For the equilateral $A B C$, the negative eddy-viscosity instability was shown to stop at a second critical Reynolds number $\operatorname{Re}_{S}^{c} \simeq 13$, where the flow becomes unstable to small-scale perturbations. For values of Re larger than $R e_{S}^{c}$ the growth-rate remains finite and independent of $q$ even at the $q \rightarrow 0$ limit. On the contrary, the fraction of energy at the largest scale becomes dependent on $q$ decreasing as $E_{0} / E_{t o t} \propto q^{4}$ in the $q \rightarrow 0$ limit. These behavior is well described by a two-modes model that is explained in sec. IIIC3. This model also predicts that in the case of an $A K A$ instability the ratio $E_{0} / E_{\text {tot }}$ scales like $E_{0} / E_{t o t} \propto q^{2}$ for $R e>R e_{S}^{c}$. This scaling was indeed found by examining the large-scale instability of a turbulent $\mathrm{ABC}$ flow, indicating that a turbulent equilateral $A B C$ is $A K A$-unstable.

Our study was carried out further to the non-linear regime where it was shown that in the presence of scale separation, the forcing scale and the largest scales of the system are the most dominant energetically. The persistence of this behavior at larger values of $R e$ remains to be examined. 


\section{ACKNOWLEDGMENTS}

This work was granted access to the HPC resources of MesoPSL financed by the Region Ile de France and the project Equip@Meso (reference ANR-10-EQPX-29-01) of the programme Investissements d'Avenir supervised by the Agence Nationale pour la Recherche and the HPC resources of GENCI-TGCC-CURIE \& GENCI-CINESJADE (Project No. x20162a7620) where the present numerical simulations have been performed.

\section{APPENDIX: FLASH}

A pseudo-spectral method is adopted to compute numerically eq. (8) and (9). The linear term are computed in Fourier space. All the terms involving the driving flow are computed in physical space made incompressible by solving in periodic space the Poisson problem, using:

$$
\boldsymbol{\Psi}^{(2)}=-\Delta^{-1}(\nabla \times)^{2} \Psi^{(1)} .
$$

The main steps of the algorithm are written below. In this algorithm, $\mathcal{F}$ and $\mathcal{F}^{-1}$ denote direct and inverse fast Fourier transforms. $\boldsymbol{A} \boldsymbol{U} \boldsymbol{X}^{(1)}$ and $\boldsymbol{A} \boldsymbol{U} \boldsymbol{X}^{(2)}$ are two aux- iliary vector fields. $\boldsymbol{A} \boldsymbol{U} \boldsymbol{X}^{(1)}$ is real and $\boldsymbol{A} \boldsymbol{U} \boldsymbol{X}^{(2)}$ is complex.

$\overline{\text { Floquet Linear Analysis of Spectral Hydrodynamic (FLASH) }}$

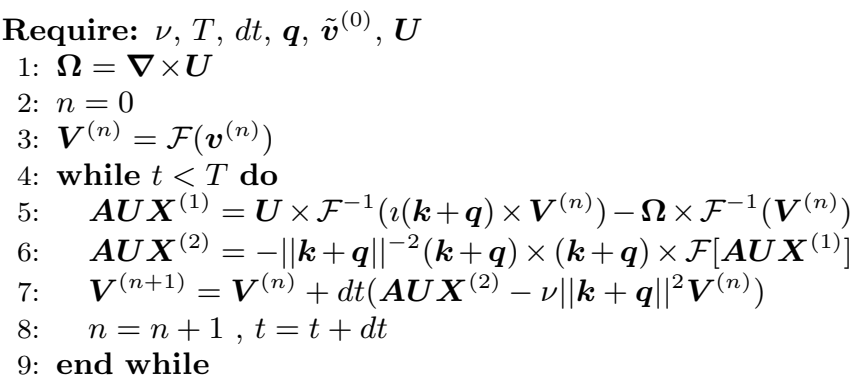

To carry out the computations with greater precision, a fourth order Runge-Kutta method is used instead of the simple Euler method at line 7 of the algorithm. The Fourier parallel expansions are also truncated at $1 / 3$ to avoid aliasing error. The code is parallelised with MPI and uses many routine from the GHOST code [23]. Most of the DNS are done at a $32^{3}$ and $64^{3}$ resolution. Convergence tests show that this resolution is sufficient for the range of Reynolds number studied.
[1] M. Steenbeck, F. Krause, and K.-H. Rädler, Zeitschrift für Naturforschung A 21, 369 (1966).

[2] H. K. Moffatt, Field Generation in Electrically Conducting Fluids (Cambridge University Press, Cambridge, London, New York, Melbourne, 1978).

[3] U. Frisch, Turbulence: The Legacy of A. N. Kolmogorov (Cambridge University Press, 1995).

[4] U. Frisch, in Turbulence and Predictability in Geophysical Fluid Dynamics and Climate Dynamics, edited by M. Gil, R. Benzi, and G. Parisi (Elsevier, Amsterdam, NorthHolland, 1985) pp. 71-88.

[5] S. G. G. Prasath, S. Fauve, and M. Brachet, EPL (Europhysics Letters) 106, 29002 (2014)

[6] T. Lee, Quart Appl Math 10, 69 (1952).

[7] S. Orszag, J. Fluid Mech. 41 (1970).

[8] R. H. Kraichnan, J. Fluid Mech 59, 745 (1973).

[9] G. Krstulovic, P. D. Mininni, M. E. Brachet, and A. Pouquet, Phys. Rev. E 79, 056304 (2009)

[10] V. Dallas, S. Fauve, and A. Alexakis, Phys. Rev. Lett. 115, 204501 (2015).

[11] U. Frisch, Z. S. She, and P. L. Sulem, Physica D: Nonlinear Phenomena 28, 382 (1987).

[12] U. Frisch, H. Scholl, Z. S. She, and P. L. Sulem,
Fluid Dynamics Research 3, 295 (1988).

[13] R. H. Kraichnan, Journal of the Atmospheric Sciences 33, 1521 (1976

[14] B. Dubrulle and U. Frisch, Physical Review A 43, 5355 (1991).

[15] A. Wirth, S. Gama, and U. Frisch, Journal of Fluid Mechanics 288, 249 (1995)

[16] G. Floquet, Annales scientifiques de l'Ecole normale superieure 12, 47 (1883).

[17] N. W. Ashcroft and N. D. Mermin, Solid state physics (Saunders College, 1976).

[18] T. Dombre, U. Frisch, J. M. Greene, M. Hénon, A. Mehr, and A. M. Soward, Journal of Fluid Mechanics 167, 353 (1986)

[19] G. O. Roberts, Philosophical Transactions of the Royal Society of Lo

[20] O. Podvigina and A. Pouquet, Physica D: Nonlinear Phenomena 75, 471 (1994).

[21] C. Marchioro, Communications in mathematical Physics 105, 99 (1986).

[22] S. E. Jones and A. D. Gilbert, Geophysical \& Astrophysical Fluid Dynamics 108, 83 (2014).

[23] P. D. Mininni, A. Alexakis, and A. Pouquet, Physical review E 77, 036306 (2008). 